\title{
Novel rare alleles of $A B C A 1$ are exclusively associated with extreme high-density lipoprotein-cholesterol levels among the Han Chinese
}

\author{
Suwei Hu', Yanfang Zhong², Yuantao $\mathrm{Hao}^{3}$, \\ Minqi Luo ${ }^{4}$, Yan Zhou ${ }^{5}$, Hui Guo ${ }^{6}$, Weijiao \\ Liao", Desheng Wan ${ }^{8}$, Haiyun Wei ${ }^{8}$, Yueting \\ $\mathrm{Gao}^{7}$, Jinglan Shan? ${ }^{7}, \mathrm{Bin} \mathrm{Hu}^{1}$, Maj Hultén ${ }^{9}$ \\ and Yiming Wang ${ }^{1, *}$ \\ ${ }^{1}$ Department of Medical Genetics, Zhongshan \\ Medical College, Sun Yat-Sen University, \\ Guangzhou, P.R. China \\ ${ }^{2}$ Department of Prenatal Diagnosis, Guangdong \\ Women and Children Hospital and Health Institute, \\ Guangzhou, P.R. China \\ ${ }^{3}$ Department of Medical Statistics and \\ Epidemiology, School of Public Health, Sun Yat-Sen \\ University, Guangzhou, P.R. China \\ ${ }^{4}$ Department of Clinical Laboratory, The Third \\ Affiliated Hospital of Sun Yat-Sen University, \\ Guangzhou, P.R. China \\ ${ }^{5}$ Fudan University, Chinese National Human \\ Genome Centre, Shanghai, P.R. China \\ ${ }^{6}$ Department of Clinical Laboratory, Shenzhen \\ People's Hospital, Shenzhen, P.R. China \\ ${ }^{7}$ Department of Clinical Laboratory, The First \\ Affiliated Hospital of Guangzhou Medical College, \\ Guangzhou, P.R. China \\ ${ }^{8}$ Department of Clinical Laboratory, Dongguan \\ People's Hospital, Dongguan, P.R. China \\ ${ }^{9}$ Warwick Medical School, University of Warwick, \\ Coventry, UK
}

\footnotetext{
Abstract

Background: High-density lipoprotein (HDL) is a major plasma lipoprotein directly associated with cholesterol metabolism. The ATP binding cassette transporter 1 gene $(A B C A 1)$ is one of the major genes modulating plasma levels of HDL-cholesterol (HDL-C). Rare alleles of $A B C A 1$ associated with extreme HDL-C concentrations have not been previously investigated in the Chinese.

Methods: Blood samples were collected from 470 subjects whose HDL-C concentrations were within the top $5 \%$ of the distribution, 335 subjects in the lowest $5 \%$, and 220 within the range 5\%-95\%. First, we sequenced all exons of the $A B C A 1$ gene from 50 sub-

*Corresponding author: Prof. Yiming Wang, Department of Medical Genetics, Zhongshan Medical College, Sun YatSen University, 74 Zhongshan Road II, Guangzhou 510089, P.R. China

Phone: +86-20-87332055, Fax: +86-20-87335785,

E-mail: ywzhong@hotmail.com

Received March 22, 2009; accepted July 8, 2009;

previously published online September 11, 2009
}

jects from the group with extremely high HDL-C, and 50 from the group with extremely low HDL-C concentrations. Next, in the remaining subjects, we genotyped the non-synonymous variants identified exclusively with either extreme group.

Results: Four novel non-synonymous alleles were identified; all were rare. Alleles c.3029C $>$ T (p.Ala1010Val) and c.5399A $>$ G (p.Asn1800Ser) were found exclusively in the low group, c.2031C $>A$ (p.Asp677Glu) and c.2660G > T (p.Cys887Phe) exclusively in the high group.

Conclusions: Our results show that some rare alleles of $A B C A 1$ are associated with marked phenotypes, supporting the "rare-variant common-disease" hypothesis. Certain alleles also provide tools for identifying individuals at high risk of dyslipidaemia, allowing for early therapeutic intervention.

Clin Chem Lab Med 2009;47:1239-45.

Keywords: ATP binding cassette transporter 1 gene $(A B C A 1)$; cholesterol efflux regulatory protein; highdensity lipoprotein cholesterol; rare allele.

\section{Introduction}

High-density lipoprotein (HDL) is one of the major plasma lipoproteins directly associated with cholesterol metabolism. Low concentrations of HDL-cholesterol (HDL-C) are a strong and independent risk factor for coronary heart disease (1). About $50 \%$ of the variation in plasma level HDL-C is believed to be genetically determined $(2,3)$. ATP binding cassette transporter 1 gene ( $A B C A$ 1, Genbank ID NM_005502, alias: $A B C 1 ; C E R P ; A B C-1)$ is one of the major genes modulating plasma HDL-C concentrations, and underlies two genetic diseases with low HDL-C: Tangier disease (MIM 205400) and familial HDL deficiency (MIM 604091) (4). ABCA1 codes for cholesterol efflux regulatory protein (CERP, UniProtKB/Swiss-Prot 095477). This protein mediates the intracellular cholesterol pump, thereby transporting cellular cholesterol to the plasma membrane and incorporating it into plasma HDL particles.

The "common-variant common-disease" is the accepted hypothesis for the genetic contribution to common disease. Recently, several studies have shown that rare alleles also contribute to common diseases, and may have stronger phenotypic effects than common alleles (5-10). This has prompted efforts to identify rare alleles in common diseases. The importance of studying rare alleles lies not only 
in identifying individuals at high risk to allow for early intervention, but also in understanding disease mechanisms and gene function. Variations in $A B C A 1$ from white and black Americans, and Danish individuals have been reported $(5,6)$. Wang and co-workers (11) screened for $A B C A 1$ mutations in a Caucasian family with Tangier disease, aboriginal Canadians of OjiCree and Inuit origin with familial HDL deficiency and a subgroup of 37 Chinese normolipidaemic subjects selected from 223 subjects. They reported three common non-synonymous variants of $A B C A 1$ : p.Val825lle, p.lle883Met and p.Arg1587Lys (originally reported as $\mathrm{V} / \mathrm{I765}, \mathrm{I} / \mathrm{M} 823$ and $\mathrm{R} / \mathrm{K} 1527$ ) among the Chinese subjects (11). However, rare alleles of the gene associated with extreme HDL-C concentrations among the Han Chinese have not been previously investigated. It is not known whether there are population-specific alleles bearing strong phenotypic effects. In an attempt to further test the "rare-variant common-disease" hypothesis and study whether there are some rare alleles that may have strong phenotypic effects among the Han Chinese, we studied subjects showing extreme HDL-C concentrations and their associated rare alleles.

\section{Materials and methods}

\section{Study subjects}

A total of 1025 subjects were recruited from the general population and individuals who underwent health checks from April to November 2008 at laboratories in two centres (the Third Affiliated Hospital of Sun Yat-Sen University and Dongguan People's Hospital) in Guangdong Province, Southern China. All subjects were of Han Chinese ethnicity. The distribution of HDL-C concentrations among the Han Chinese and selection for genotyping were based on distribution data for Japanese subjects recommended by Daiichi Pure Chemicals (male $1.41 \pm 0.33 \mathrm{mmol} / \mathrm{L}$, female $1.58 \pm$ $0.32 \mathrm{mmol} / \mathrm{L}$; http://www.sekisuimedical.jp/). These ranges were adopted at the two centres used in this study. The study group included 470 subjects, in whom HDL-C concentrations were in the top $5 \%$ of the distribution (extremely high group); 335 in the lowest $5 \%$ (extremely low group); and 220 subjects in which HDL-C was within the 5\% and 95\% limits (middle-range group). Individuals with diseases or conditions that may affect plasma HDL-C, such as diabetes mellitus, dysthyroidism, liver dysfunction, and pregnancy, were excluded. All subjects gave informed consent. The study was conducted in accordance with the Declaration of Helsinki and was approved by the University Ethics Committee.

\section{HDL-C measurement}

Following a 12-h fast, blood was collected in tubes that promote coagulation. Blood samples were centrifuged at $1800 \mathrm{~g}$ for $10 \mathrm{~min}$ and the supernatant carefully separated. HDL-C was measured using a homogenous method (12), the Cholestest N HDL kit (Daiichi Pure Chemicals, Kyoto, Japan) with a Hitachi autoanalyzer (Hitachi, Tokyo, Japan) according to the manufacturer's instructions. Cholestest $\mathrm{N}$ calibrator (Daiichi Pure Chemicals) was used to calibrate the assay necessary. Liquid Assayed Multiqual (BioRad, Irvine, CA, USA) was used for quality control to monitor the precision of the HDL-C procedure. The quality control value for HDL-C for Level 2 was $1.39 \pm 0.15 \mathrm{mmol} / \mathrm{L}$, and for Level 3, $1.97 \pm$ $0.15 \mathrm{mmol} / \mathrm{L}$.

\section{Detection of rare alleles of $A B C A 1$}

Direct sequencing of all exons in $\mathbf{5 0}$ subjects in the high and 50 subjects in the low HDL-C groups As phenotype-associated rare alleles are more likely to be harboured in extreme phenotypes, we first explored rare alleles in the low and high HDL-C groups. Fifty randomly selected subjects (31 males and 19 females, age $55 \pm 18$ years) in the low HDL-C group and 50 subjects ( 28 males and 22 females, age $46 \pm 19$ years) in the high HDL-C group were first screened for $A B C A 1$ variation. Genomic DNA was extracted from peripheral blood cells with Tiangen DNA blood Mini kits (Tiangen, Beijing, China) according to the manufacturer's instructions. Primers were designed for all the exons and intron-exon boundaries, with 100-200 bp extensions into intronic regions. PCR products were sequenced in both directions using the $A B I$ Sequence Analyzer $3730 \times \mathrm{L}$ (Applied Biosystems, Foster City, CA, USA). Results were compared with sequences retrieved from the UCSC Genome Browser (http://genome. ucsc.edu). Standard nomenclature, as described by den Dunnen and Antonarakis (www.hgvs.org/mutnomen/) (13), was used for description of DNA sequence variation, with +1 corresponding to the $A$ of the ATG translation initiation codon of the GenBank mRNA sequence NM_005502.2. Genomic DNA (gDNA) numbering is also provided with the first nucleotide of GenBank reference sequence NG_007981 as +1.

TaqMan-based genotyping assays in the rest of the assembly Six rare non-synonymous variants, not shared in both groups, were identified using direct sequencing as described above, using high and low HDL-C groups. The variants were then tested for in the remaining individuals with extreme HDL-C (high and low groups) using the TaqManbased genotyping assay with the ABI 7500 real-time PCR System (Applied Biosystems). In order to explore the phenotypic effects and further confirm the rarity of these alleles, we also tested for these same alleles in the subjects with HDL-C in the middle-range. Primer Express 2.0 (Applied Biosystems) was used to design primers and probes (listed in Table 1). Premix Ex TaqTM kit (TaKaRa, Otsu, Shiga, Japan) was used in the genotyping assay. The reaction mix contained $10 \mu \mathrm{L}$ premix, $10 \mu \mathrm{M}$ primers, $10 \mu \mathrm{M}$ probes, $0.4 \mu \mathrm{L}$ ROX reference dye, and $10 \mathrm{ng}$ DNA template, in a total volume of $20 \mu \mathrm{L}$. Amplification was carried out with one $10 \mathrm{~s}$ cycle at $95^{\circ} \mathrm{C}, 50,5 \mathrm{~s}$ cycles at $95^{\circ} \mathrm{C}$ and maintenance at $60^{\circ} \mathrm{C}$ for $34 \mathrm{~s}$. The SDS v1.2× System Software (Applied Biosystems) was used to analyse the results. Sample genotypes confirmed by direct sequencing were used as positive controls and samples with no DNA as negative controls in each plate.

\section{Cross-species alignment of $A B C A 1$ cDNA and protein sequences}

The CLUSTAL X (1.81) program (http://www.clustal.org/) was used to compare the human $A B C A 1$ cDNA sequence (NM_005502) with nucleotide sequences of the mouse (Mus musculus) (NM_013454.3), rat (Rattus norvegicus) (NM_178095.2) and chicken (Gallus gallus) (NM_204145.1). The same program was used to compare amino acid sequences between these species (human UniProtKB/SwissProt 095477; mouse P41233; rat Q80ZB2; and chicken Q8UVV4). 
Table 1 Primers and probes used in the TaqMan-based genotyping assay.

\begin{tabular}{|c|c|c|c|}
\hline Variations & Substitutions & Primer/probe & Sequence $5^{\prime} \rightarrow 3^{\prime}$ \\
\hline p.Arg496Trp & c. $1486 \mathrm{C}>\mathrm{T}$ & $\begin{array}{l}\text { Primers-F/R } \\
\text { Probe-C } \\
\text { Probe-T }\end{array}$ & $\begin{array}{l}\text { TCCAGTCCAGTAATGGTTCTGTGTA/CCTGTGTTAGGCTTGAGGGATAGT } \\
\text { FAM-CTAACCAGGCAATCCGGACCATATCTC-TAMRA } \\
\text { HEX-CTAACCAGGCAATCTGGACCATATCTCG-TAMRA }\end{array}$ \\
\hline p.Asp677Glu & c. $2031 \mathrm{C}>\mathrm{A}$ & $\begin{array}{l}\text { Primers-F/R } \\
\text { Probe-C } \\
\text { Probe-A }\end{array}$ & $\begin{array}{l}\text { TCACAAGAAGAGGAATGAGGCTACT/CTTTCTCCCCTTGTCCTTCCTT } \\
\text { FAM-ATGCTGTTGTCCAGGCCCATGAT-TAMRA } \\
\text { HEX-ATGCTGTTTTCCAGGCCCATGATC-TAMRA }\end{array}$ \\
\hline p.Cys887Phe & c. $2660 \mathrm{G}>\mathrm{T}$ & $\begin{array}{l}\text { Primers-F/R } \\
\text { Probe-G } \\
\text { Probe-T }\end{array}$ & $\begin{array}{l}\text { GTGCTTTCTGTGGGTTCATTTCT/ACCTTCATCCCATCTCGGTAGA } \\
\text { FAM-TCCTGTCACAGTCTGCATGGAGGAG-TAMRA } \\
\text { HEX-CCTGTCACAGTCTTCATGGAGGAGGA-TAMRA }\end{array}$ \\
\hline p.Ala1010Val & c. $3029 \mathrm{C}>\mathrm{T}$ & $\begin{array}{l}\text { Primers-F/R } \\
\text { Probe-C } \\
\text { Probe-T }\end{array}$ & $\begin{array}{l}\text { TTTTGCTTTTCAGCTTGCTTGA/AGGCTGACTGTCGAAGAACACAT } \\
\text { FAM-TCCATCTCCGCCTTCACGTGC-TAMRA } \\
\text { HEX-CTGCTCCATCTCCACCTTCACGTG-TAMRA }\end{array}$ \\
\hline p.Val1096lle & c. $3286 \mathrm{G}>\mathrm{A}$ & $\begin{array}{l}\text { Primers-F/R } \\
\text { Probe-G } \\
\text { Probe-A }\end{array}$ & $\begin{array}{l}\text { GAGGAGCCCACACAGCACA/TCCAGTGCTTACCCCTGCTAAT } \\
\text { FAM-CCCCAGGACGTCCGCTTCATC-TAMRA } \\
\text { HEX-CCCAGGATGTCCGCTTCATCCA-TAMRA }\end{array}$ \\
\hline p.Asn1800Ser & c.5399A $>$ G & $\begin{array}{l}\text { Primers-F/R } \\
\text { Probe-A } \\
\text { Probe-G }\end{array}$ & $\begin{array}{l}\text { ATGAATGACACCCGTTTCTTCTC/ATTGCCTGGTTTTTCACCATGT } \\
\text { FAM-AATAATATCAATGATATCCTGAAGT-MGB } \\
\text { HEX-ATAATATCAGTGATATCCTGAAG-MGB }\end{array}$ \\
\hline
\end{tabular}

Topological model of wild-type and mutant proteins

HMMTOP (14), TMHMM (15), TMpred (16), Tmap (17), DAS (18), Phobius (19), TOPpred (20) and SOSUI (21) softwares were used to model the transmembrane topology of the wild-type and mutant CERP proteins found to be exclusively associated with extreme HDL-C concentrations.

\section{Results}

\section{HDL-C levels}

A total of 335 subjects with extremely low HDL-C, 470 with extremely high HDL-C concentrations and 220 with middle-range HDL-C were recruited. Details of HDL-C concentrations, gender and age of the subjects are shown in Table 2.

\section{Rare alleles identified and their association with HDL-C levels}

Results of direct sequencing in the $\mathbf{5 0}$ high and $\mathbf{5 0}$ low HDL-C subjects A total of 21 variants were discovered using direct sequencing of the randomly selected subjects in the two groups with extreme HDL-C concentrations (Table 3), 17 coding and four non-coding. Among the coding variants, 11 were non-synonymous and six synonymous with respect to amino acid sequence; five were novel. Six of the 11 non-synonymous variants were identified exclusively in either the low or the high HDL-C groups, the other five were shared by these two groups (Table 3 ). The six exclusive alleles were rare in the subjects who were sequenced (Table 3 ).

Results of the TaqMan-based genotyping assays and comparisons between the different HDL-C groups Genotyping of the six exclusive alleles in the remaining subjects comprising the groups with high and low $\mathrm{HDL}-\mathrm{C}$, and the subjects with HDL-C in the middle range (middle-range HDL-C group), further confirmed the rarity of the six alleles (Table 4). The c.1486C $>T$ (p.Arg496Trp) allele initially identified in the low HDL-C group only, was also found in the middle HDL-C group. Allele c.3286G $>$ A (p.Val1096Ile), initially identified only in the high HDL-C group, was alsofj found in the middle HDL-C group. Therefore, the alleles associated exclusively with high HDL-C were c.2031C $>$ A (p.Asp677Glu) and c.2660G $>$ T (p.Cys887Phe). The alleles associated exclusively with low HDL-C were c.3029C $>$ T (p.Ala1010Val) and c.5399A $>$ G (p.Asn1800Ser) (Table 4, Figure 1). These four exclusive alleles are all novel (Table 4).

\section{Across-species comparisons}

Alleles c.2031C $>$ A (p.Asp677Glu), c.2660G $>$ T (p.Cys887Phe) and c.5399A $>$ G (p.Asn1800Ser) occurred in the highly conserved nucleotide and

Table 2 Plasma concentrations of HDL-C, gender and age of the study population.

\begin{tabular}{|c|c|c|c|c|c|c|}
\hline & \multicolumn{2}{|l|}{ Low HDL-C } & \multicolumn{2}{|l|}{ High HDL-C } & \multicolumn{2}{|c|}{ Middle-range HDL-C } \\
\hline & Male & Female & Male & Female & Male & Female \\
\hline $\mathrm{HDL}-\mathrm{C}, \mathrm{mmol} / \mathrm{L}$ & $0.60 \pm 0.17$ & $0.72 \pm 0.18$ & $2.25 \pm 0.26$ & $2.39 \pm 0.23$ & $1.39 \pm 0.14$ & $1.64 \pm 0.15$ \\
\hline Age, years & $53 \pm 18$ & $52 \pm 19$ & $47 \pm 16$ & $54 \pm 14$ & $51 \pm 15$ & $47 \pm 12$ \\
\hline Number & 192 & 143 & 290 & 180 & 91 & 129 \\
\hline Total number & \multicolumn{2}{|c|}{335} & \multicolumn{2}{|c|}{470} & \multicolumn{2}{|c|}{220} \\
\hline
\end{tabular}


Table 3 Variants of $A B C A 1$ identified by direct sequencing.

\begin{tabular}{|c|c|c|c|c|c|c|c|}
\hline \multirow[t]{3}{*}{ Gene region } & \multirow[t]{3}{*}{ Nucleotide change } & \multirow[t]{3}{*}{ Amino acid } & \multirow{3}{*}{$\begin{array}{l}\text { References or } \\
\text { dbSNP ID }\end{array}$} & \multicolumn{4}{|c|}{ No. of subjects } \\
\hline & & & & \multicolumn{2}{|c|}{ Low $(n=50)$} & \multicolumn{2}{|c|}{ High $(n=50)$} \\
\hline & & & & $\mathrm{H}^{\mathrm{a}}$ & $\mathrm{Q}^{\mathrm{b}}$ & $\mathrm{H}^{\mathrm{a}}$ & $\mathrm{Q}^{\mathrm{b}}$ \\
\hline \multicolumn{8}{|c|}{ Untranslated exonic region } \\
\hline Exon 2 & g.24399_24400insG & - & rs1799777 & 11 & 0 & 10 & 0 \\
\hline Exon 2 & g. $24459 \bar{G}>C$ & - & rs1800978 & 11 & 0 & 10 & 0 \\
\hline Exon 50 & g. $146152 A>G$ & - & rs4149341 & 27 & 2 & 24 & 4 \\
\hline Exon 50 & g.146738_146740delGTT & - & rs41474449 & 23 & 0 & 20 & 0 \\
\hline \multicolumn{8}{|c|}{ Translated exonic region } \\
\hline \multicolumn{8}{|c|}{ Non-synonymous } \\
\hline Exon 7 & c.656G $>A$ & p.Arg219Lys & rs2230806 & 24 & 7 & 39 & 1 \\
\hline Exon 12 & c. $1486 C>T$ & p.Arg496Trp & Ref. (5) & 1 & 0 & 0 & 0 \\
\hline Exon 15 & c.2031C $>$ A & p.Asp677Glu & Novel & 0 & 0 & 1 & 0 \\
\hline Exon 16 & c. $2311 \mathrm{G}>\mathrm{A}$ & p.Val771Met & rs2066718 & 5 & 0 & 2 & 0 \\
\hline Exon 17 & c. $2473 \mathrm{G}>\mathrm{A}$ & p.Val825lle & rs2066715 & 25 & 7 & 37 & 4 \\
\hline Exon 18 & c. $2649 \mathrm{G}>\mathrm{A}$ & p.Met883Ile & rs2066714 & 19 & 7 & 19 & 3 \\
\hline Exon 19 & c. $2660 \mathrm{G}>\mathrm{T}$ & p.Cys887Phe & Novel & 0 & 0 & 1 & 0 \\
\hline Exon 21 & $c .3029 \mathrm{C}>\mathrm{T}$ & p.Ala1010Val & Novel & 1 & 0 & 0 & 0 \\
\hline Exon 23 & c.3286G $>$ A & p.Val1096Ile & rs13306073 & 0 & 0 & 1 & 0 \\
\hline Exon 35 & c. $4760 \mathrm{G}>\mathrm{A}$ & p.Arg1587Lys & rs2230808 & 24 & 6 & 31 & 4 \\
\hline Exon 40 & c. $5399 \mathrm{~A}>\mathbf{G}$ & p.Asn 1800Ser & Novel & 1 & 0 & 0 & 0 \\
\hline \multicolumn{8}{|c|}{ Synonymous } \\
\hline Exon 6 & c. $474 \mathrm{G}>\mathrm{A}$ & p.Leu158 & rs2230805 & 13 & 16 & 13 & 21 \\
\hline Exon 9 & c. $936 \mathrm{C}>\mathrm{T}$ & p.Pro312 & rs2274873 & 1 & 0 & 6 & 0 \\
\hline Exon 9 & c. $948 \mathrm{G}>\mathrm{A}$ & p.Gly316 & rs2246841 & 3 & 0 & 2 & 0 \\
\hline Exon 15 & c. $2040 \mathrm{~A}>\mathrm{C}$ & p.lle680 & rs2853579 & 19 & 7 & 20 & 3 \\
\hline Exon 16 & c. $2148 \mathrm{C}>\mathrm{T}$ & p.Ser716 & Novel & 0 & 0 & 1 & 0 \\
\hline Exon 31 & c. $4281 \mathrm{G}>\mathrm{A}$ & p.Thr1427 & rs2066716 & 22 & 8 & 22 & 7 \\
\hline
\end{tabular}

${ }^{a} \mathrm{H}$, heterozygote of the minor alleles; ${ }^{b} \mathrm{Q}$, homozygote of the minor allele. Rare variants associated with extreme $\mathrm{HDL}-\mathrm{C}$ concentrations are indicated in bold font.

amino acid sequences in all four species studied. Nucleotide C in c.3029C $>$ T (p.Ala1010Val) was conserved in humans, mice and rats, but not in chickens (Figure 2).

\section{Transmembrane topology of wild-type and mutant proteins}

Gene $A B C A 1$ contains 50 exons, translation of CERP being initiated in exon 2 , terminating in exon 50 (http://genome.ucsc.edu). The wild-type protein has two membrane-integral domains, each containing six membrane-spanning $\alpha$-helices and two ATP-hydrolysing domains (22). Transmembrane topology simulation showed no predictable differences between wild-type and mutant proteins. However, all the exclusively-associated alleles were located in the functionally important regions, implying potential modes of action of these alleles. These are p.Asp677Glu in the first intracellular loop; p.Cys887Phe in the seventh

Table 4 Rare alleles identified in the whole study population.

\begin{tabular}{|c|c|c|c|c|}
\hline \multirow[t]{2}{*}{ Allele } & \multirow{2}{*}{$\begin{array}{l}\mathrm{HDL}-\mathrm{C}, \mathrm{mmol} / \mathrm{L} \\
\text { (Male/female) }\end{array}$} & \multicolumn{2}{|c|}{ No. of subjects ${ }^{a}$} & \multirow[t]{2}{*}{ Domain } \\
\hline & & $\begin{array}{l}\text { In this } \\
\text { group }\end{array}$ & $\begin{array}{l}\text { In the whole } \\
\text { assembly } \\
(n=1025)\end{array}$ & \\
\hline Low HDL-C group & & $\mathrm{n}=335$ & & \\
\hline c.3029C > T (p.Ala1010Val) & $0.74 /-^{\mathrm{b}}$ & 1 & 1 & 1st $A B C^{c}$ \\
\hline c.5399A > G (p.Asn1800Ser) & $-{ }^{b} / 0.74$ & 1 & 1 & 5th intracellular loop \\
\hline High HDL-C group & & $\mathrm{n}=470$ & & \\
\hline c.2031C >A (p.Asp677Glu) & $-\mathrm{b} / 2.13$ & 1 & 1 & 1st intracellular loop \\
\hline c.2660G > T (p.Cys887Phe) & $2.09 / 2.27$ & 2 & 2 & $H 7^{\mathrm{d}}$ \\
\hline Low HDL-C group + middle group & & $\mathrm{n}=555$ & & \\
\hline c.1486C > T (p.Arg496Trp) & $-\mathrm{b} / 1.03 \pm 0.79$ & 2 & 2 & Extracellular $N$-term loop \\
\hline High HDL-C group + middle group & & $\mathrm{n}=690$ & & \\
\hline c.3286G > A (p.Val1096lle) & $2.14 \pm 0.06 / 2.23 \pm 0.21$ & 6 & 6 & 1st $A B C^{c}$ \\
\hline $\begin{array}{l}\text { Other combinations of HDL-C groups } \\
\text { No rare variation }\end{array}$ & & & & \\
\hline
\end{tabular}

${ }^{a}$ All subjects were heterozygotes of the minor allele; ${ }^{b}$ no rare variation was found with respect to gender of the subject; ${ }^{\circ} A B C$, ATP-hydrolysing domain; ${ }^{\mathrm{d}} \mathrm{H} 7$, the 7th hydrophobic segment; ${ }^{\mathrm{e}}$ other combinations of HDL-C groups includes the "high" +"low" and "high" +"low + middle" groups. 


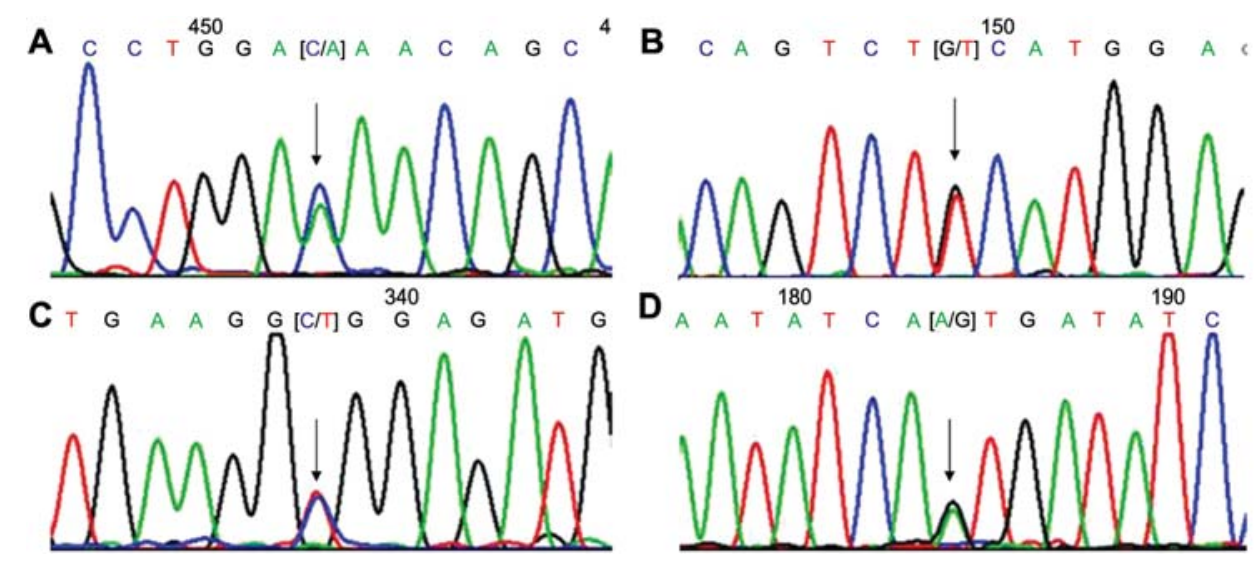

Figure 1 Partial sequencing results of the forward strand of the four rare alleles.

Arrows denote the alleles. (A) c.2031C $>$ A; (B) c.2660G > T; (C) c.3029C $>$ T; (D) c.5399A $>$ G.

hydrophobic segment; p.Ala1010Val in the first ATPhydrolysing domain, known to be necessary for transporter activity (23), and p.Asn1800Ser in the fifth intracellular loop.

\section{Discussion}

The $A B C A 1$ gene belongs to the large $A B C$ membrane transporter superfamily. The encoded proteins transport various molecules, including metabolites, cholesterol, steroids and drugs across intra- and extra-cellular membranes. In humans, there are 49 paralogues of the superfamily, and 13 of these have been identified as being responsible for Mendelian diseases, including cystic fibrosis (MIM 219700), Startgardt disease (MIM 600110), Dubin-Johnson syndrome (MIM 237500), progressive familial intrahepatic cholestasis-3 (MIM 602347), X-linked sideroblastic anaemia and ataxia (MIM 301310). The ABCA1 encoded protein, CERP, modulates cholesterol metabolism by mediating the "reverse transport" of cholesterol by pumping intracellular cholesterol to the plasma membrane and its subsequent incorporation into plasma HDL particles. The liver is the major organ where cholesterol is degraded and secreted. ABCA1, therefore, plays a crucial role in regulating plasma cholesterol concentrations. This has been clearly demonstrated in Tangier disease (MIM 205400) and familial HDL deficiency (MIM 604091). The classical phenotypes of Tangier disease are the near absence of HDL-C in plasma and clinical symptoms caused by intracellular cholesterol accumulation including enlarged yellow tonsils, splenohepatomegaly, and peripheral neuropathy (24). In familial HDL deficiency, plasma HDL-C is greatly reduced, accompanied by a higher incidence of premature coronary heart disease and atherosclerosis (25).

Plasma HDL-C concentrations are determined by both genetic and environmental factors. Inheritance is generally considered to be polygenic in the majority of subjects, but, as stated above, there are also Mendelian conditions associated with extreme HDL-C concentrations like Tangier disease and familial HDL deficiency. Cohen and colleagues demonstrated that rare alleles of $A B C A 1$ are associated with extreme HDL-C concentrations in Caucasians and African Americans in the US (5). This was confirmed by a large population-based study of subjects from Denmark that showed that both rare alleles and common variants also contribute to HDL-C concentrations in the general population (6). These and other recent studies (5-10) demonstrate that rare alleles not only contribute to common diseases, but can also exert stronger phenotypic effects than common variants. To prioritise our efforts on the strong phenotype-bearing alleles, the present study was restricted to identify rare alleles. Our results show that alleles of c.3029C $>$ T (p.Ala1010Val) and c.5399A $>$ G (p.Asn1800Ser) are exclusively associated with low HDL-C, c.2031C $>$ A (p.Asp677Glu) and c.2660G $>$ T (p.Cys887Phe) with high HDL-C in Han Chinese. These alleles were rare, but the frequencies obtained from the whole assembly may not be representative of true population frequencies. This is because there were more subjects tested in the extreme HDL-C groups than in the middle-range group, and the ratio of males and females was not equivalent. The three common non-synonymous variants reported by Wang et al. (11) were also detected in our subjects, but none was associated exclusively with extreme HDL-C concentrations. These results not only support the "rare-variant common-disease" hypothesis, but also demonstrate that rare alleles can have strong phenotypic effects in different populations. The four extreme phenotype-associated alleles are novel. More importantly from a clinical point of view, our results provide a novel tool for identifying individuals at high risk, with the potential for early intervention. In the present study, allele c.1486C > T (p.Arg496Trp), previously shown to be associated with high HDL-C in American subjects, was identified in Han Chinese subjects from the groups with extremely low- and middle-range HDL-C concentrations. The reason for this discrepancy is currently unknown and further studies are needed to address this question.

The $A B C A 1$ gene is located on $9 q 31$ and consists of 50 exons. Its protein product is translated from within 
A

Homo sapiens

Mus musculus

Rattus norvegicus

Gallus gallus

Homo sapiens

Mus musculus

Rattus norvegicus Galilus gallus

Homo sapiens

Mus musculus Rattus norvegicus Gallius gallus

Homo sapiens Mus musculus Rattus norvegicus Gallius gallus

Homo sapiens Mus musculus Rattus norvegicus Gâlilus gallus

\section{Homo sapiens Mus musculus Rattus norvegicu Gallius gallus}

\section{D}

Homo sapiens Mus musculus Rattus norvegicus Gallius gallus

Homo sapiens Nus musculus Rattus norvegicus Garlius gallus c. $2031 \mathrm{C}>\mathrm{A}$ CTGAAGAGACCATGCGGATCATGGGCCTGGAGMACAGCATCCTCTGGTTTAG CTGAAGGAGACCATGCOSATCATGGOTCTGGACAATGGCATCCTCTGGTTTAG TTGAAGGAGACCATGCGCATCATGGGCCTGGACATGGCATCCTCTGGTTTAG CTGAAGGAACAATGAOGATTATGGGCCTTGAC ATGGCATCCTTTGGCTAAG

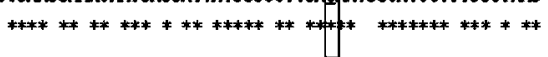
D. Asp677Glu

I IKG IVYEKEARLKETMRIMGI DISILWF SWF ISSLIPLLVSAGL LVILKLG I IRS IVYEKRARL KETMRIMGLONGILWF SWFVSLLIPLLVSAGLLVVILKLG I IKSIVTEKEARLKETTRIMGL DVGILWF SWF ISSLIPILVSAGLLVIILKLG I TKG IVYEKEARIKETMRIMGI ONGILWLSWF ISSLIPLLLSAGLIVLILKMG

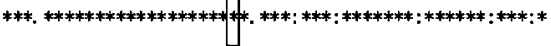
c. $2660 \bigcirc>T$

CCAACCAGAAGAGAATATCAGAATCTCCATGGAGGACGAACCCACCCACTIG CCAGCCAGAAGGGAGTGTCAGAATCTCEATGGAAGAGGAACCCACTCATCTG CCAGOCAGAAGGGTGCGTCAGAAATCICEATGGAAGAGGAGCCCACTCACCTG CTGACCAGAAGCOCCCCTCAGAAGTCICCAAGGAGGAAGAGCCTATGCATCTG

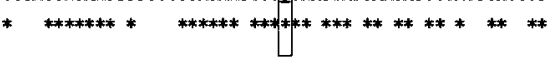
p.Cys887Phe

PCTKSYWFGEESTEKSHPGSNQKR ISE ICYFEEPTHLKLGVS IQNLVKVTRDG PCTKSYWFGEE IDEKSHPGSSQRGUSE ICUEREPTHLRLGVSIONLVKVYRDG PCTKSYWFGEE IDEKSTFGSSQKG ASE ICWEEEPTHLKLGVSIONLYKVYRDG

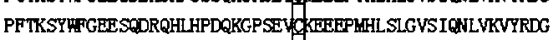

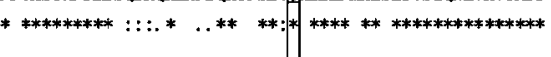
c. $3029 \mathrm{C}>\mathrm{T}$

CACGTGAAGO OSGAGATGGAGCAGATGGCOCTGGATGTTGG TTTGCCATCAA CACGTGAANG OGSGATGGAGCAGATGG CCCTGGATGTTCGCTTACCCCCOA CATGTGAAACCAGAGATGGAGCAGATGGCCCTGGATGTTGGCTTACCOCCCA AAGGTAAAAGAOCGAAATGGAGCAGATCGCAATCGACGTTCGTTTCCCTC--AAGGTAAAGG GCAAATGGAGCAGATCGCAATCGACGTTCGTTTCCCTC

\section{p.Ala1010Val}

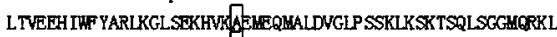
LTVEHIWF TARLKGLSEKIVNAEMEQMALDNGIPPSKLKSKTSQLSGGMQRKL LTVEEH IWF YARLKGLSEKHYM QENEQMALNGLPPSKLKSKTSQLSGGHCRKL LTVEEH IUF YARLKGLPEKKVKIODWEQHAHDWGLP-HKLKAR TSKLSCGMCRKL

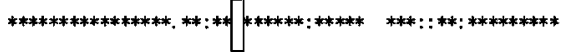
c. $5399 \mathrm{~A}>\mathrm{G}$

GGAGCTGTTCACOGACAATAAGCTGAATAATATCAQTGATATCCTGAAGTCOGT GGAGCTGTT TACAAACAATAGGTCAATGACATCA STGACATCCTGAAGTCTGT GGAGCTCTTTACAAACAAT TAATTCAATGACATCA S TGACATCCTGAAATCTGT GGAGCTCTTCACCAACAACAAGCTGAACAACATCA QTGACATCTTGAATTCTGT

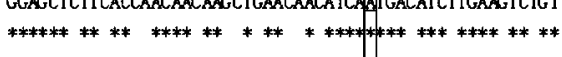
p.Asn1800Ser

LTSWLF IGINGSVATFVEIFTDNKLNNISDI IKSWF LIFPHFCLGRGLIDMVK LTSWNLF IGINGSVATF VIELFTNNKLADINDI LKSYFL IFPHHCLCRGLIDWVK LTSWLF IGINGSVATFVELFTNNKFNDINDI LKSWFLIPPHFCLCRGLIDHVK LTSWLF IGINGSVA TTVIELFTRWKLNNINDI LKSWF LIFPHFCLCRGLIDWVK ************************;**:*:***************************

Figure 2 Multiple sequence alignment of $A B C A 1$ across species.

The CLUSTAL X (1.81) program was used to align the cDNA and protein sequences between species. The nucleotides and amino acids corresponding to the four rare variants are framed. (A) c.2031C $>$ A (p.Asp677Glu); (B) c.2660G $>$ T (p.Cys887Phe); (C) c.3029C > T (p.Ala1010Val); (D) c.5399A > G (p.Asn1800Ser). Identical bases and amino acids in the four species are denoted with asterisks.

exon 2 and terminates in the part of exon 50 that consists of 2261 amino acids (http://www.uniprot.org/ uniprot/). Topological models of wild-type and mutant proteins show that all the extreme phenotype-associated mutant sites occur in functionally important regions. None occurred in the transmembrane section, consistent with previous findings in a Danish population (6). Rare alleles are important, although the present study demonstrates that not all rare alleles have significant phenotypic effects. In the present study, alleles c.1486C $>$ T (p.Arg496Trp) and c.3286G $>$ A (p.Val1096lle) were not associated with any phenotypic changes. Some rare alleles, even coding variations, may therefore be neutral, as also found in the Danish population (6).

Plasma HDL-C concentrations and studies of the genetic contributions underlie that it serves as a good example to show that most clinical conditions can be caused by mutations in more than one gene, as well as by a whole spectrum of frequencies of alleles that may be common or rare within each gene. Some rare alleles are more often associated with extreme phenotypes. Re-sequencing and deep-sequencing efforts are therefore important for revealing these rare but significant alleles. This is important not only for identification of individuals at high risk who may benefit from early intervention, but also for our basic understanding of mechanisms of disease.

\section{Acknowledgements}

The authors thank all the volunteers who donated blood samples for this study. This work was supported by the 985 Project of China, Phase II; the Natural Science Foundation of Guangdong Province (Grant No. 031673) and the Guangzhou Municipal Science and Technology Foundation (Grant No. 2002Z3-C7191 and 2004Z3-C7501).

\section{References}

1. Gotto AM Jr. High-density lipoprotein cholesterol and triglycerides as therapeutic targets for preventing and treating coronary artery disease. Am Heart J 2002;144: S33-42.

2. Friedlander Y, Kark JD, Stein Y. Biological and environmental sources of variation in plasma lipids and lipoproteins: the Jerusalem Lipid Research Clinic. Hum Hered 1986;36:143-53.

3. Hunt SC, Hasstedt SJ, Kuida H, Stults BM, Hopkins PN, Williams RR. Genetic heritability and common environmental components of resting and stressed blood pressures, lipids, and body mass index in Utah pedigrees and twins. Am J Epidemiol 1989;129:625-38.

4. Brooks-Wilson A, Marcil M, Clee SM, Zhang LH, Roomp $\mathrm{K}$, van Dam M, et al. Mutations in $\mathrm{ABC} 1$ in Tangier disease and familial high-density lipoprotein deficiency. Nat Genet 1999;22:336-45.

5. Cohen JC, Kiss RS, Pertsemlidis A, Marcel YL, McPherson $\mathrm{R}$, Hobbs HH. Multiple rare alleles contribute to low plasma levels of HDL cholesterol. Science 2004;305:86972.

6. Frikke-Schmidt R, Nordestgaard BG, Jensen GB, TybjaergHansen A. Genetic variation in $A B C$ transporter A1 contributes to $\mathrm{HDL}$ cholesterol in the general population. $J$ Clin Invest 2004;114:1343-53.

7. Fearnhead NS, Wilding JL, Winney B, Tonks S, Bartlett S, Bicknell DC, et al. Multiple rare variants in different genes account for multifactorial inherited susceptibility to colorectal adenomas. Proc Natl Acad Sci USA 2004;101: 15992-7.

8. Cohen JC, Pertsemlidis A, Fahmi S, Esmail S, Vega GL, Grundy SM, et al. Multiple rare variants in NPC1L1 associated with reduced sterol absorption and plasma lowdensity lipoprotein levels. Proc Natl Acad Sci USA 2006; 103:1810-5.

9. Walsh T, McClellan JM, McCarthy SE, Addington AM, Pierce SB, Cooper GM, et al. Rare structural variants 
disrupt multiple genes in neurodevelopmental pathways in schizophrenia. Science 2008;320:539-43.

10. Nejentsev S, Walker N, Riches D, Egholm M, Todd JA. Rare variants of IFIH1, a gene implicated in antiviral responses, protect against type 1 diabetes. Science 2009;324:387-9.

11. Wang J, Burnett JR, Near S, Young K, Zinman B, Hanley $\mathrm{AJ}$, et al. Common and rare ABCA1 variants affecting plasma HDL cholesterol. Arterioscler Thromb Vasc Biol 2000;20:1983-9.

12. Warnick GR, Nauck M, Rifai N. Evolution of methods for measurement of HDL-cholesterol: from ultracentrifugation to homogeneous assays. Clin Chem 2001;47:1579-96.

13. den Dunnen JT, Antonarakis SE. Mutation nomenclature extensions and suggestions to describe complex mutations: a discussion. Hum Mutat 2000;15:7-12.

14. Tusnady GE, Simon I. The HMMTOP transmembrane topology prediction server. Bioinformatics 2001;17:84950.

15. Sonnhammer EL, von Heijne G, Krogh A. A hidden Markov model for predicting transmembrane helices in protein sequences. Proc Int Conf Intell Syst Mol Biol 1998; 6:175-82.

16. Hofmann K, Stoffel W. TMBase - a database of membrane spanning protein segments. Biol Chem Hoppe-Seyler 1993;374:166.

17. Milpetz F, Argos P, Persson B. TMAP: a new email and WWW service for membrane-protein structural predictions. Trends Biochem Sci 1995;20:204-5.
18. Cserzo M, Wallin E, Simon I, von Heijne G, Elofsson A. Prediction of transmembrane alpha-helices in prokaryotic membrane proteins: the dense alignment surface method. Protein Eng 1997;10:673-6.

19. Kall L, Krogh A, Sonnhammer EL. A combined transmembrane topology and signal peptide prediction method. J Mol Biol 2004;338:1027-36.

20. Claros MG, von Heijne G. TopPred II: an improved software for membrane protein structure predictions. Comput Appl Biosci 1994;10:685-6.

21. Hirokawa T, Boon-Chieng S, Mitaku S. SOSUI: classification and secondary structure prediction system for membrane proteins. Bioinformatics 1998;14:378-9.

22. Fitzgerald ML, Morris AL, Rhee JS, Andersson LP, Men$\operatorname{dez} A J$, Freeman MW. Naturally occurring mutations in the largest extracellular loops of ABCA1 can disrupt its direct interaction with apolipoprotein A-I. J Biol Chem 2002;277:33178-87.

23. Marcil M, Brooks-Wilson A, Clee SM, Roomp K, Zhang $\mathrm{LH}, \mathrm{Yu} \mathrm{L}$, et al. Mutations in the $\mathrm{ABC} 1$ gene in familial HDL deficiency with defective cholesterol efflux. Lancet 1999;354:1341-6.

24. Assmann G, von Eckardstein A, Brewer HB. Familial HDL deficiency: Tangier disease. In: Scriver CR, Beaudet AL, Sly WS, Valle D, editors. The metabolic basis of inherited disease. New York: McGraw-Hill, 1995:2053-72.

25. Miller NE. Associations of high-density lipoprotein subclasses and apolipoproteins with ischemic heart disease and coronary atherosclerosis. Am Heart J 1987;113: 589-97. 